
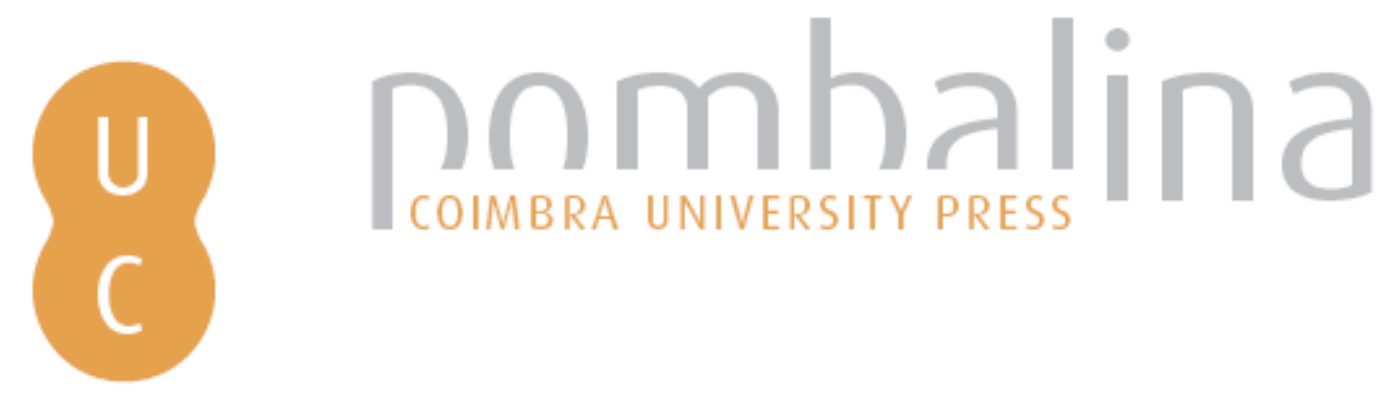

\title{
Le Baccanti di Euripide: un'opera post-moderna?
}

Autor(es): $\quad$ Greco, Giovanni

Publicado por: Centro de Estudos Clássicos e Humanísticos da Universidade de

Coimbra; Imprensa da Universidade de Coimbra

URL

persistente: URI:http://hdl.handle.net/10316.2/30244

DOI: $\quad$ DOI:http://dx.doi.org/10.14195/978-989-721-038-9_26

Accessed : $\quad$ 26-Apr-2023 16:28:22

A navegação consulta e descarregamento dos títulos inseridos nas Bibliotecas Digitais UC Digitalis, UC Pombalina e UC Impactum, pressupõem a aceitação plena e sem reservas dos Termos e Condições de Uso destas Bibliotecas Digitais, disponíveis em https://digitalis.uc.pt/pt-pt/termos.

Conforme exposto nos referidos Termos e Condições de Uso, o descarregamento de títulos de acesso restrito requer uma licença válida de autorização devendo o utilizador aceder ao(s) documento(s) a partir de um endereço de IP da instituição detentora da supramencionada licença.

Ao utilizador é apenas permitido o descarregamento para uso pessoal, pelo que o emprego do(s) título(s) descarregado(s) para outro fim, designadamente comercial, carece de autorização do respetivo autor ou editor da obra.

Na medida em que todas as obras da UC Digitalis se encontram protegidas pelo Código do Direito de Autor e Direitos Conexos e demais legislação aplicável, toda a cópia, parcial ou total, deste documento, nos casos em que é legalmente admitida, deverá conter ou fazer-se acompanhar por este aviso.

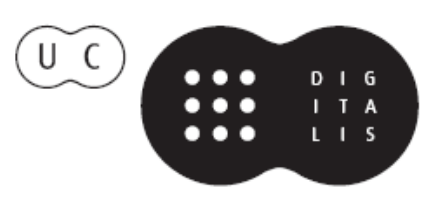




\section{De ayer a hoy}

\section{Influencias clásicas en la literatura}

\section{Aurora López, Andrés Pociña, Maria de Fátima Silva (coords.)}




\section{LE BACCANTI DI EURIPIDE: UN'OPERA POST-MODERNA?}

Giovanni Greco

Theatron - Universitá di Roma La Sapienza

Le Baccanti sono un testo enigmatico, sincretistico, all'insegna della contaminazione: la storia di Dioniso e di Penteo ha molti punti di contatto con quella di Cristo; la condizione delle menadi, al seguito di Bacco, è molto simile a quella delle tarantolate, donne che nel profondo Sud dell'Italia (Puglia), hanno vissuto per secoli una trance dionisiaca, conseguenza del morso della taranta, mitico ragno, emblema della loro repressione sessuale, che le portava a danzare e cantare per giorni, al fine di scioglere le pulsioni pericolose; infine, la vicenda di Penteo, che vuole spiare la madre sui monti mentre compie ta orgia, sembra alludere al voyeurismo della scena primaria freudiana: il desiderio edipico di Penteo si realizza, ma in un senso macabro -potrà tornare nel grembo materno, ma fatto a pezzi, regredire ad un inizio che è fine, nel compimento di un principio di piacere che coincide con la pulsione di morte.

Una delle scene più famose della storia del teatro inizia al v. 912 delle Baccanti di Euripide. Penteo, giovane re di Tebe, fiero avversario di Dioniso e dell'introduzione del suo culto 'effeminato' a Tebe, rientra in scena travestito da donna e afferma di vedere 'due soli e due volte Tebe dalle sette porte'. Come è stato possibile che il persecutore della 'buona novella' dionisiaca sia diventato all'improvviso, nelle parole se non nei comportamenti, icona di Bacco?

Penteo ha osteggiato con tutte le forze lo Straniero, ovvero Dioniso che ha assunto sembianze umane come straniero che viene dall'Oriente; ha lottato contro le donne del suo seguito che hanno invaso Tebe e fatto proseliti: tra queste la madre di Penteo stesso, Agave, e le sue sorelle, Ino e Autonoe, che ora si trovano, in trance sul Citerone, a compiere ta orgia. Penteo ha fatto catturare lo Straniero effeminato, lo ha umiliato e lo ha sbattuto in carcere, ma quest'ultimo si è liberato dalle catene e la sua liberazione è coincisa con un terremoto straordinario che ha scosso la reggia alle fondamenta (vv. 576-604). Una volta libero, Dioniso-Straniero si è fatto beffe di Penteo, lo ha contro-umiliato (v. 616), facendolo correre dietro a fantasmi ed inganni, portandolo sull'orlo dell'esasperazione e della follia (vv. 616-641: lo racconta lo stesso Straniero alle donne del Coro terrorizzate dopo il terremoto). Esasperazione che tocca il suo culmine, quando, uscendo fuori dal palazzo, esausto di correre dietro alle illusioni dionisiache, Penteo trova Dioniso-Straniero libero e ascolta le parole del messaggero di ritorno dal Citerone, che racconta delle imprese mirabolanti delle donne sul monte (vv. 677-774). È a seguito di quest'ultimo racconto, che l'offensiva del re Penteo contro Dioniso e il dionisismo, si trasforma, prende una 
direzione inaspettata: la polemica di Penteo contro l'irrazionalismo del culto bacchico trova, grazie alle seduzioni dello Straniero e alle parole 'incredibili' del Messaggero, una nuova via di fuga. Penteo decide o crede di decidere, dopo qualche ultima resistenza, che sarà lui in persona a risolvere la 'vergogna' delle Baccanti sul Citerone: andrà lui sul monte, vestito da baccante 'per non farsi riconoscere', a spiare cosa fanno davvero le donne e metterà fine alle loro orgia. Entra dunque dentro il palazzo per prepararsi, cioè travestirsi da menade e muovere verso le donne, accompagnato da Dioniso-Straniero, che questa soluzione gli ha, subdolamente, suggerito (vv. 768-846).

Quando rientra in scena, al già citato v. 912, Penteo, che ha ormai l'aspetto di Dioniso-Straniero, dà l'impressione di vederci doppio o di essere sotto effetto di sostanze psicotrope, di essere ubriaco o addirittura impazzito, comunque preda dell'invasamento dionisiaco. Vestito con un peplo bianco, la cintura da donna, acconciato con i riccioli biondi della maschera dionisiaca e con in mano il tirso, freme di lanciarsi verso il Citerone, accompagnato da suo cugino Dioniso, che, come un bravo regista, perfeziona gli ultimi dettagli della messa in scena e lo rassicura sull'esito positivo del suo viaggio al monte: il dio del teatro traveste con le sue stesse fattezze l'iniziando, cioè la futura vittima sacrificale, nella cornice di una scena metateatrale che avrà una fortuna enorme, in forme più o meno esplicite, per tutta la storia del teatro. Questo perché la storia del teatro è, strutturalmente, storia en travestie e non si dà teatro senza mascheramento, scambio d'identità, ambiguità di genere, equivoco. $\grave{E}$ in questa scena delle Baccanti che si presenta, per la prima volta e in forma tragicomica, quella commedia degli errori o degli equivoci che da Euripide arriverà a Menandro e per il tramite di Plauto e Terenzio, attraverso Shakespeare irromperà nel teatro moderno e persino nel cinema. Le Baccanti, opera metateatrale che mette in scena il dio del teatro nel suo teatro, indaga e mette in mostra il meccanismo profondo del teatro stesso, il mistero della finzione e della convenzione teatrale, la dialettica inesauribile di straniamento e immedesimazione (Verfremdungeffekt e Einfüblung) tra attore e personaggio e lo fa in uno snodo storico cruciale come il 405-404 a. C.: la fine della Guerra del Peloponneso, la crisi della democrazia ateniese, la fine di un mondo e l'inizio di un altro. Tutto questo non sembra essere un caso.

La domanda è: cos'è che ha reso così fortunata questa scena? Cos'è che ce la fa piacere, che ci seduce, cioè ci fa ridere, fremere, forse piangere al contempo? A me pare si possa dire che questa scena sia fondativa di quella drammaturgia definibile come edipica, cioè di quella drammaturgia che realizza, nel suo intreccio, il complesso di Edipo, anche se non ne è direttamente consapevole e anche se il suo punto di vista è a- o anti-edipico nel senso freudiano, esattamente come sostenevano Vernant e Vidal-Naquet ormai quarant'anni fa. Se è vero che l'Edipo re di Sofocle ha poco o nulla a che fare con quello che 
Freud desume nell'Interpretazione dei sogni a partire dai vv. 980-982 dell'Edipo $r$, ciò non toglie che la drammaturgia tragica e comica post-classica (latina, rinascimentale, del Siglo de oro, etc.) sia profondamente familistica, tutta centrata sulla dialettica tra patri-monio e matri-monio, dedita con poche, significative eccezioni, all'infinita variazione sul tema del desiderio di uccidere il padre e giacere con la madre che da Edipo arriva a Amleto e da Amleto fino ai nostri giorni (in Italia, per esempio, all'incesto mancato dei Sei personaggi in cerca d'autore di Pirandello). La 'per-versione' del desiderio totalizzante del bambino che vuole essere e avere tutto all'interno delle mura domestiche, è tema che convive con altri per tutto il $\mathrm{V}$ secolo a. C. (per esempio il tema del dolore del corpo o quello dell'amicizia tradita o anche quello della sfida agli dèi), ma che, nelle mutate condizioni politiche, sociali ed economiche, vince e si impone a partire dal IV sec. a. C., in una casistica sterminata e ossessiva: nelle Baccanti la declinazione sosfisticata del complesso di Edipo è quella che, freudianamente, si definisce della 'scena primaria'. La scena primaria è, nella vulgata freudiana, uno dei paradigmi ineludibili nello sviluppo della personalità e nella costruzione dell'identità: il bambino vede concretamente, immagina o simbolizza l'evento topico dei genitori che fanno l'amore e, nelle multiple possibilità di attraversamento di quell'evento, prefigura la sua futura omosessualità o eterosessualità, le sue nevrosi, le sue psicosi, le sue perversioni voyeuristiche, etc. L'urgenza di Penteo di spiare la madre Agave e le sorelle di lei che compiono ta orgia sul monte (sono consapevole del fatto che orgia ha un significato non solo erotico ma anche sacrale), rappresenta una forma particolare, si potrebbe dire sado-masochistica, della scena primaria in assenza di padre, la cui conclusione, tragica, sarà, per il giovane re di Tebe, il ritorno al grembo materno, la regressio ad uterum dopo essere stato mangiato e bevuto dalla madre e dalle sorelle di lei, in una straordinaria coincidenza tra principio di piacere e pulsione di morte, che, per dirla ancora con Freud, va da Penteo alla madre e viceversa. Il racconto del secondo messaggero, di ritorno dal Citerone (vv. 1043-1152), mette in scena il desiderio finalmente realizzato del figlio fatto a pezzi, sacrificato e infine ritornato nel ventre materno come eucaristia, fractio panis e libagione del vino, dio mangiato nel corpo e nel sangue del suo iniziando.

Sì. Proprio eucaristia. Perché la conclusione della vicenda di Penteo in quanto figlio, 'crocifisso' all'abete sul Citerone, è una conclusione cristologica, l'ultimo atto di una mitopoiesi dionisiaca che intrattiene diversi punti di contatto con quella cristiana e in realtà con molte mitopoiesi orientali (si pensi soltanto a quella di Iside e Osiride). Anzi, Dioniso è diaframma inesauribile tra Oriente e Occidente (cfr. la parodo, in questo senso, vero e proprio evangelo dionisiaco, vv. 64-167) e, non a caso, le Baccanti sono l'opera con cui un anonimo autore medievale del XII secolo d. C. ha composto un'opera 
dal titolo Christus patiens che, centonando i versi di Euripide, racconta la passione, la morte e la resurrezione di Cristo. Noi ricostruiamo, infatti, una parte considerevole della lacuna che si apre al v. 1329 delle Baccanti (circa 50 versi), e cioè tutta la compositio membrorum e la lamentatio che segue al ritorno sulla scena di Agave ancora in trance con la testa del figlio infilzata al tirso (simbolo fallico) e che lentamente riprende coscienza, rendendosi conto di quanto ha fatto (vv. 1168 e ss.), proprio grazie al confronto con il Christus patiens. Le Baccanti sono, significativamente, costellate di eventi che rimandano alla passione di Cristo e alla sua vicenda umana e divina: dal terremoto con cui 'muore' e 'resuscita' lo Straniero (vv. 576-603, per cui si veda Matteo 27,51-53), al taglio della testa di Penteo, riportato in scena da e per una donna (la testa di Penteo, cugino di Dioniso, ricorda quella di Giovanni Battista, cugino di Gesù, riportata su un vassoio a Salomè). In qualche modo, la macabra comunione fisica che si realizza tra madre e figlio nel finale delle Baccanti, in senso freudiano, parrebbe rimandare, mutatis mutandis, alla compenetrazione tutta spirituale e sublimata tra la mater dolorosa che piange sotto la croce e poi al sepolcro e il figlio morto fatto a pezzi, crocifisso e offerto in sacrificio della tradizione cristiana. Nel primo caso, senza redenzione; nel secondo caso, per la salvezza dell'umanità.

L'eroe tragico (o comico), figura sempre replicata del canone occidentale, originerebbe, dunque, al contatto tra una humus edipica, sulle cui radici nel folclore di tutti i tempi ha scritto pagine insuperate Vladimir Propp, e la declinazione cristiana di questa humus, su cui pagine insuperate ha scritto invece Jan Kott proprio in relazione alle Baccanti. Questo eroe rimane, nel corso delle successive rivisitazioni, teatrali e letterarie, un eroe trionfante e sconfitto, un eroe dibattuto, che è, insieme e alternativamente, tyrannos e pharmakòs, re e 'crocifisso', che tocca il vertice dell'onore e quello dell'abominio, il più venerato e il più reietto di tutti gli uomini: l'eroe dell'ambiguità. Gli esempi sono molteplici, anche quando la forma che questa scena e il suo eroe o i suoi eroi assumono, li rende quasi irriconoscibili rispetto al punto di partenza. Mi limito ad un esempio vicinissimo a noi nel tempo, epoca di revival delle Baccanti euripidee e di una certa declinazione sincretica del dionisiaco, inteso non in senso nietzscheano come antitesi dell'apollineo, ma come sinonimo dell'identità ambigua, incompiuta, nomade e di una convivenza post-moderna degli opposti, che vuol dire meticciato, multidisciplinarietà, sottolineatura della dimensione ludica e performativa delle relazioni umane (persino paradigma di una tolleranza religiosa neopagana, rispetto al monoteismo tradizionale).

La ricerca filosofica più recente parla, in questo senso, dell'identità come gioco, come messa in scena, come performance: la stessa identità biologica non sarebbe un fatto naturale ma culturale, travestimento, maschera; il proprio 
genere (addirittura il proprio numero) non è dato una volta per tutte, ma giocato, recitato di volta in volta nel tempo e nello spazio, barattato e (ri)costruito perché l'essere è un ibrido che riesce per intervalla insaniae a stabilire una prevalenza, ma non sa imporla a se stesso e al mondo una volta per sempre. Cadono, nell'universo concettuale postmoderno e nelle sue pratiche, le distinzioni, i confini tra umano e animale, tra uomo e macchina, tra natura e cultura in senso più generale: si parla di post-umano, post-organico, si parla di corpo senz'organi (Artaud), ciò che rimodula o abolisce categorie politiche, etiche, estetiche. Ecco allora, tra le forme più estreme e dionisiache del postmoderno, le performance della body-art, che non propongono più all'analisi critica l'opera d'arte come oggetto prodotto da un soggetto, ma che fanno del corpo del soggetto stesso unopera d'arte, sezionandolo, ricostruendolo, estendendolo o riducendolo fino a renderlo equivoco, 'mostruoso', demonico ${ }^{1}$, nell'indistinzione tra vita e arte, tra etica e estetica. Alla base, seppure dimenticata o trasfigurata, resta, a mio parere, la scena madre o scena madre-figlio in assenza di padre da cui abbiamo cominciato:

\section{Dioniso}

Tu che vuoi vedere quel che non si deve, cercare quel che non si cerca, tu Penteo, esci dal palazzo, lasciati guardare, quel tuo vestitino da menade folle, 915 ci vai a spiare tua madre e il suo codazzo: hai le forme di una delle figlie di Cadmo.

Penteo

Ho l'impressione di vedere due soli, $e$ due volte Tebe, dalle sette porte. $E$ tu che mi stai davanti sembri un toro, ti sono spuntate le corna sulla testa. Ma tu eri una belva un tempo? Ora sei un toro...

\section{Dioniso}

Dio è con noi, non ci voleva bene prima, ora è ben disposto: e vedi quel che devi.

Penteo

E come ti sembro? Ho l'anadatura di Ino, o magari quella di Agave, mia madre?

${ }^{1}$ Il versante comico-parodico di questa 'contaminazione' è Tootsie per il cinema, con Dustin Hoffmann; per quello letterario Creatura di sabbia di T. B. Jelloun; per il teatro Attempts to her life di Martin Crimp. 
Dioniso

Ti guardo e mi sembra di vedere loro.

Ma ti è andato un ricciolo fuori di posto,

non come l'avero messo io sotto il nastro.

Penteo

È che prima dentro a casa, e su e giù, io, a fare

la tua danza, mè uscito fuori posto.

\section{Dioniso}

Ma io, che ho solo cuore di essere il tuo schiavo, te lo tirerò su: forza alza la testa.

Penteo

Ecco, fammi bello: sono nelle tue mani.

Dioniso

La cintura s'è allentata, e le pieghette del vestito non scendono alle caviglie.

\section{Penteo}

Sembra anche a me, ma solo qui al piede destro, dall'altro mi pare scenda a perfezione.

Dioniso

Non mi farai il primo dei tuoi amici, quando le vedrai virtuose a dispetto di te?

Penteo

Il tirso lo prendo con la destra o l'altra

per assomigliare di più a una baccante?

Dioniso

Con la destra devi prenderlo ed il piede destro alzare: bravo, stai perdendo i freni

Penteo

E ce la farei a portare sulle spalle

il Citerone con tutte le baccanti?

\section{Dioniso}

Ce la fai, se vuoi: prima eri un po' frenato, ora invece stai bene, come si deve. 
Penteo

Portiamo le leve? O tiro su le vette

con le mani e me le metto in spalla o in braccio?

Dioniso

Ma distruggerai le case delle Ninfe,

le sedi di Pan dove i flauti risuonano.

Penteo

Ben detto; bisogna vincere le donne

senza forza: io tra gli abeti mi nascondo.

Dioniso

Ti nasconderai come s'ba da nascondere

uno che va a spiare ingannando le menadi.

Penteo

Secondo me stanno tra i cespugli come

uccelli presi nelle reti d'amore.

Dioniso

E non è per questo che vai ad esplorare?

Le prenderai se non sarai preso prima.

Penteo

Portami attraverso la terra di Tebe:

io sono di tutti l'unico a osar questo.

Dioniso

L'unico a soffrire per la città, l'unico:

ci sono le prove ad aspettarti debite.

Seguimi: tua scorta sono io tua salvezza,

ti riporterà un altro da lì.

Penteo

La madre?

Dioniso

L'esempio per tutti.

Penteo

Eper questo ci vado.

Dioniso

Portato verrai... 
Giovanni Greco

Penteo

Dici la mia delizia.

Dioniso

Tra mani di madre.

Penteo

Mi forzi a godere.

Dioniso

E che godimenti.

Penteo

Quelli che mi merito.

Dioniso

Tremendo tremendo vai alla tua passione tremenda, una gloria troverai fino al cielo. Allunga le mani, Agave, e voi che siete del seme di Cadmo: io porto il ragazzino alla grande prova; chi vincerà, io, sarà Bromio. Il resto significherà. 